\title{
Effect of Nanostructures on Heat Transfer Coefficient of an Evaporating Meniscus
}

\author{
Han $\mathrm{Hu}$ and Ying Sun ${ }^{*}$ \\ Department of Mechanical Engineering and Mechanics, Drexel University, Philadelphia, PA \\ 19104, USA
}

\begin{abstract}
The effect of nanostructures on heat transfer coefficient of an evaporating meniscus in thin film evaporation and nucleate boiling is investigated using combined modeling and molecular dynamics (MD) simulations. The model is developed accounting for the evaporation kinetics, disjoining pressure, conduction resistance, and Kapitza resistance of an evaporating meniscus on a nanostructured surface. The model is then verified using MD simulations for a water-gold system with square nanostructures of varying depth and film thickness. Good agreement is obtained between MD results and model predictions. The results show the existence of a critical film thickness on the order of a few nanometers where the heat transfer coefficient reaches its maximum. For a film thickness below this critical value, the evaporation resistance dominates and the heat transfer coefficient increases with film thickness but decreases with nanostructure depth due to the enhanced disjoining pressure. However, for a film thickness greater than the critical value, the conduction resistance dominates and the heat transfer coefficient decreases with film thickness but increases with nanostructure depth. In addition, both critical film thickness and maximum heat transfer coefficient increase with the roughness ratio of the nanostructure, mainly due to the reduction in Kapitza resistance.
\end{abstract}

*Corresponding author. Tel.:+1-215-895-1373; fax: +1-215-895-1478; Email: ysun@coe.drexel.edu. 
Keywords: Thin film evaporation; Nucleate boiling; Nanostructures; Disjoining pressure; Kapitza resistance; Molecular dynamics 


\section{Introduction}

Thermal management is becoming a severe bottleneck in ever-increasing demand toward miniaturization of high performance integrated circuits, power electronics, and lasers. Phase change through boiling is one of the most effective heat transfer mechanisms by utilizing the high latent heat of vaporization to dissipate thermal energy, with the maximum theoretical heat flux on the order of $10,000 \mathrm{~W} / \mathrm{cm}^{2}[1]$. However, there exists a practical limit of boiling heat flux, the critical heat flux (CHF), above which the rapid development of an insulating vapor layer blankets the heat transfer surface. Based on the Helmholtz instability of vapor columns leaving a solid surface, Zuber predicted the CHF of water on a planar surface to be $\sim 110 \mathrm{~W} / \mathrm{cm}^{2}$, which agrees well with pool boiling experiments utilizing planar surfaces [2].

Recent efforts using micro/nanostructured and hierarchical structured surfaces to enhance heat transfer coefficient (HTC) and CHF of boiling experiments have resulted in enhanced CHF on the order of 200-313 W/cm ${ }^{2}[1,3-14]$. This enhancement in CHF can be attributed to a variety of mechanisms, including increased nucleation site densities [1, 5, 15], elongated contact line $[7,16]$, enhanced micro-convection around nucleated bubbles $[4,17,18]$, increased bubble departure frequency [19], and enhanced microlayer evaporation via a strong wicking effect [3, 9, 13, 14, 20-22]. Among these possible mechanisms, the enhanced microlayer evaporation through wicking has been widely accepted as the dominant mechanism, where correlations $[3,21]$ and theoretical predictions [22] have been established between the CHF and the wicking rate. For a bubble nucleated on a solid surface during boiling, evaporation occurs both along the apparent liquid-vapor interface and across the thin film (or microlayer) near the bubble contact line as shown in Fig. 1a, where nanostructure-enhanced liquid delivery through wicking stabilizes the evaporating thin film, thereby improving the CHF. In order to better understand nanostructure- 
enhanced boiling heat transfer, the effect of the nanostructures on the heat transfer coefficient of an evaporating thin film needs to be systematically studied.

The evaporating meniscus near a contact line can be divided into three regions: the nonevaporating film region, evaporating thin film region, and intrinsic meniscus region, as illustrated in Fig. 1b. In the evaporating thin film region, due to the presence of solid-liquid intermolecular forces, an excess pressure known as the disjoining pressure is required for liquid molecules to escape from the thin film. This disjoining pressure increases rapidly with decreasing liquid film thickness and its gradient, induced by the change in liquid film thickness of the evaporating meniscus, drives the liquid to flow from the intrinsic meniscus to the evaporating thin film region such that a high heat flux in the thin film region is maintained. Modeling of heat transfer in an evaporating thin film was pioneered by Wayner et al. [23], where the role of disjoining pressure in both liquid delivery and evaporation suppression was identified. A thin film evaporation model was further developed to include the effects of slip boundary [24], thermocapillary [24, 25], electrostatic on disjoining pressure [25], heat conduction through thin film [25-27], and capillary suppression [27, 28]. Wang et al. [27] analyzed the evaporation of a meniscus in nanochannels and found that the evaporating thin film region (from the nonevaporating film to the location of film thickness $\sim 1 \mu \mathrm{m}$ ) accounts for more than $50 \%$ of the total heat transfer. Narayanan et al. [25] showed that the electrostatic disjoining pressure elongates the thin film region and increases the total evaporation rate. Despite their proven success, to the best of authors' knowledge, existing thin film evaporation models have been developed based on planar surfaces where the effects of nanostructures on disjoining pressure, capillary pressure, heat conduction, and thus the overall heat transfer performance are not accounted for. 
(a)

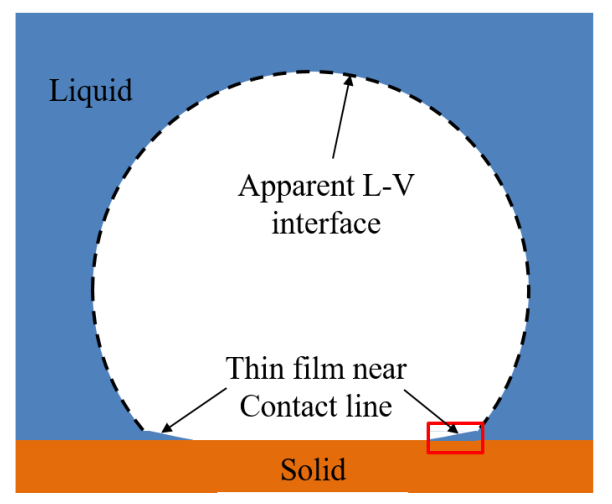

(b)

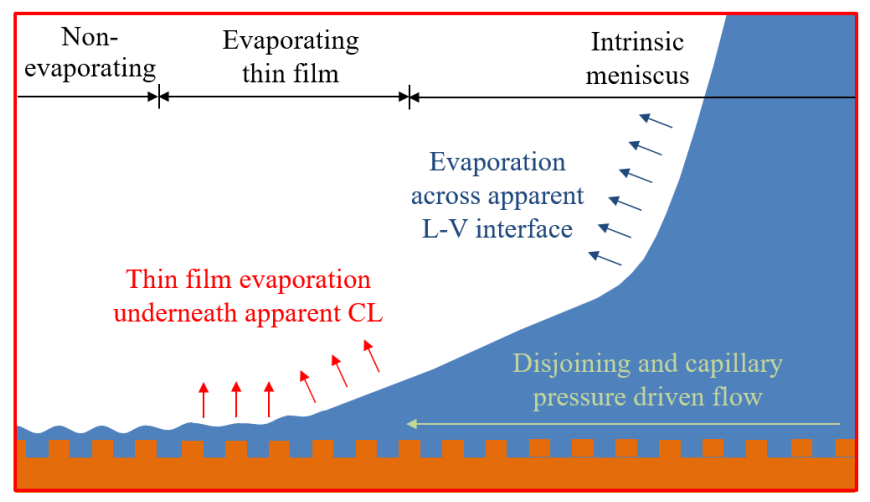

Figure 1. (a) Schematic of a bubble in nucleate boiling. (b) Zoomed-in schematic of the contact line region of the bubble on a nanostructured surface.

Recently, $\mathrm{Hu}$ et al. developed a closed-form model to account for the effect of nanostructures on disjoining pressure $[29,30]$. However, how the nanostructure-enhanced disjoining pressure affects the heat transfer rate of an evaporating thin film has not been systematically investigated. Furthermore, existing thin film evaporation models neglected the Kapitza resistance, i.e., the interfacial thermal resistance caused by the mismatch in thermal properties between two contact materials. It is important to note that the Kapitza resistance at a water-gold interface is $\sim 10^{-8} \mathrm{~m}^{2} \mathrm{~K} / \mathrm{W}[31,32]$, equivalent to the conduction resistance of a $10 \mathrm{~nm}$ water film. As the non-evaporating water film becomes as thin as a few nanometers [25], the Kapitza resistance, which is significantly affected by nanostructures, becomes nontrivial [32, 33]. Molecular dynamic (MD) simulations have become a powerful tool to investigate nanoscale heat transfer, including disjoining pressure [34] and Kapitza resistance [32]. However, atomistic simulations are limited by the specific material systems and geometries studied, making the generalization to other systems difficult. 
In the present study, a closed-form model is developed to determine the heat transfer coefficient of an evaporating meniscus on a nanostructured surface by integrating evaporation kinetics with disjoining pressure and Kapitza resistance so as to better quantify the impact of nanostructures on heat transfer performance of thin film evaporation and nucleate boiling. Molecular dynamics simulations are performed for thin water films evaporating on square nanostructured gold surfaces of varying film thickness and nanostructure depth to verify the model predictions. The effects of film thickness and nanostructure depth on heat transfer coefficient are examined. The results presented here will be used to guide design of nanostructured surfaces with a variety of materials and geometries for enhanced thin film evaporation and boiling heat transfer.

\section{The Model}

In this section, a model is developed to predict the heat transfer coefficient of a thin liquid film evaporating on a nanostructured surface by integrating evaporation kinetics, disjoining pressure, and Kapitza resistance. The schematic of a thin liquid film of thickness $\delta_{0}$ evaporating on a two-dimensional periodic nanostructure of wavelength $L$ and depth $D$ is shown in Fig. 2 using a square nanostructure as an example. The basic assumptions of the model are:

i) The van der Waals interactions are the dominating intermolecular interactions between the liquid film and the nanostructured surface.

ii) The vapor near the thin film is assumed to behave as an ideal gas.

iii) The meniscus shape, $\zeta_{\mathrm{L}}$, is periodic with the same wavelength $L$ as that of the substrate surface $\zeta_{s}$. 
iv) The thin liquid film wets the nanostructured surface in Wenzel state, where liquid is in full contact with the nanostructured surface.

v) The evaporation and condensation at the liquid-vapor interface can be determined based on evaporation kinetics.

vi) The vapor phase is not far from equilibrium. Specifically, the difference between the liquid temperature at the liquid-vapor interface, $T_{\mathrm{lv}}$, and the vapor temperature, $T_{\mathrm{v}}$, is small, i.e., $\left|T_{1 \mathrm{v}}-T_{\mathrm{v}}\right| \ll<T_{\mathrm{v}}$ and $\left|P_{\text {equ }}\left(T_{\mathrm{lv}}\right)-P_{\mathrm{v}}\left(T_{\mathrm{v}}\right)\right| \ll<P_{\mathrm{v}}\left(T_{\mathrm{v}}\right)$, where $P_{\text {equ }}\left(T_{\mathrm{lv}}\right)$ is the equilibrium pressure at the liquid-vapor interface and is different from the saturation pressure due to the presence of the disjoining and capillary pressures.

vii) The temperature of the solid surface is uniform at $T_{\mathrm{s}}$.

viii) The conduction in the liquid phase is one dimensional (along $\mathrm{z}$ direction).

Figure $2 \mathrm{~b}$ shows the resistance network model of thin film evaporation, which includes the Kapitza resistance at the solid-liquid interface, $R_{\mathrm{K}}$, the conduction resistance in the liquid film, $R_{\text {cond }}$, and the evaporation resistance at the liquid-vapor interface, $R_{\text {evap }}$. Based on the kinetic theory, the evaporative heat flux at the liquid-vapor interface is given following Schrage's derivation $[35,36]$

$$
q^{\prime \prime}=\left(\frac{2 \hat{\sigma} h_{f g}}{2-\hat{\sigma}}\right)\left(\frac{M}{2 \pi R}\right)^{1 / 2}\left[\frac{P_{\mathrm{equ}}\left(T_{\mathrm{lv}}\right)}{T_{\mathrm{lv}}^{1 / 2}}-\frac{P_{\mathrm{v}}\left(T_{\mathrm{v}}\right)}{T_{\mathrm{v}}^{1 / 2}}\right]
$$

where $\hat{\sigma}$ is the accommodation coefficient related to the liquid property, $M$ the molar mass of the liquid, and $R$ the universal gas constant. For a bulk liquid with a flat interface, $P_{\text {equ }}\left(T_{\mathrm{lv}}\right)$ equals to the saturation pressure $P_{\text {sat }}\left(T_{\mathrm{lv}}\right)$. However, for a thin liquid film on a nanostructured 
surface, the equilibrium pressure, $P_{\text {equ }}\left(T_{\text {lv }}\right)$ deviates from the saturation pressure due to the effects of the disjoining pressure, $\Pi$, and capillary pressure, $P_{\mathrm{C}}$, following [37]

$$
\ln \left(\frac{P_{\text {equ }}\left(T_{\mathrm{lv}}\right)}{P_{\text {sat }}\left(T_{\mathrm{lv}}\right)}\right)=-\frac{M}{\rho_{\mathrm{l}} R T_{\mathrm{lv}}}\left(P_{\mathrm{C}}+\Pi\right)
$$

where $\rho_{1}$ is the liquid density. Using the Clausius-Clapeyron equation to relate the vapor pressure $P_{\mathrm{v}}\left(T_{\mathrm{v}}\right)$ with the saturated pressure at $T_{\mathrm{lv}}$ following $\ln \left(P_{\text {sat }}\left(T_{\text {lv }}\right) / P_{\mathrm{v}}\left(T_{\mathrm{v}}\right)\right)=M h_{\mathrm{fg}}\left(1 / T_{\mathrm{v}}-1 / T_{\mathrm{lv}}\right) / R$ and assuming $\left|P_{\text {equ }}\left(T_{\text {lv }}\right)-P_{\mathrm{v}}\left(T_{\mathrm{v}}\right)\right| \ll<P_{\mathrm{v}}\left(T_{\mathrm{v}}\right)$,

becomes

$$
\frac{P_{\text {equ }}\left(T_{\mathrm{lv}}\right)-P_{\mathrm{v}}\left(T_{\mathrm{v}}\right)}{P_{\mathrm{v}}\left(T_{\mathrm{v}}\right)}=\frac{M h_{\mathrm{fg}}}{R}\left(\frac{1}{T_{\mathrm{v}}}-\frac{1}{T_{\mathrm{lv}}}\right)-\frac{M}{\rho_{\mathrm{l}} R T_{\mathrm{lv}}}\left(P_{\mathrm{C}}+\Pi\right)
$$

Considering the heat conduction in the liquid film and the Kapitza resistance at the solid-liquid interface, the solid surface temperature, $T_{\mathrm{s}}$, can be related to the temperature at the liquid-vapor interface, $T_{1 \mathrm{v}}$, following

$$
T_{\mathrm{s}}-T_{\mathrm{lv}}=\left(\frac{\delta^{*}}{k_{1}}+R_{\mathrm{K}}\right) q^{\prime \prime}
$$

where $k_{1}$ is the liquid conductivity, $R_{\mathrm{K}}$ is the Kapitza resistance, and $\delta^{*}$ is the modified local film thickness. Note that the local film thickness $\delta(x)$ is defined as $\delta(x)=\zeta_{\mathrm{L}}(x)-\zeta_{\mathrm{S}}(x)$ [38]. For a nanostructured surface, a modified local film thickness $\delta^{*}(x)$ is introduced to denote the shortest distance between the meniscus profile and the solid surface following $\delta^{*}(x)=\delta(x) / r$, where $r=\int_{-\frac{L}{2}}^{\frac{L}{2}} \sqrt{1+\left(\zeta_{\mathrm{S}}^{\prime}\right)^{2}} d x / L$ is the Wenzel roughness ratio between the actual and flat 
surfaces. This gives rise to a modified mean film thickness $\delta_{0}^{*}=\frac{1}{L} \int_{-\frac{L}{2}}^{\frac{L}{2}} \delta^{*}(x) d x=\delta_{0} / r$ [29], where $\delta_{0}$ is the mean film thickness.

(a)

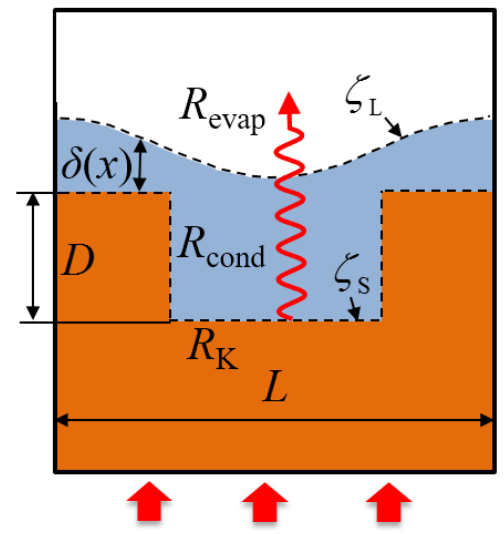

(b)

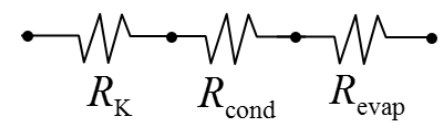

(c)

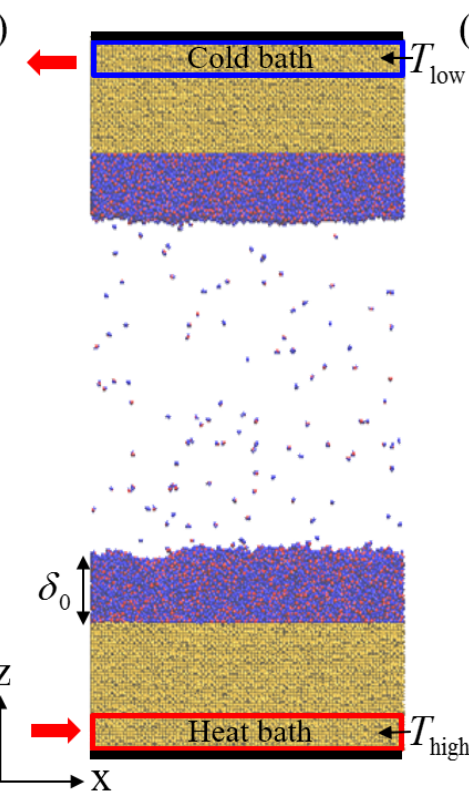

(d)
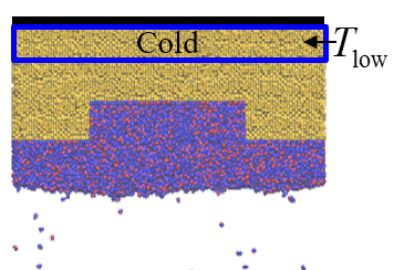

$\therefore$
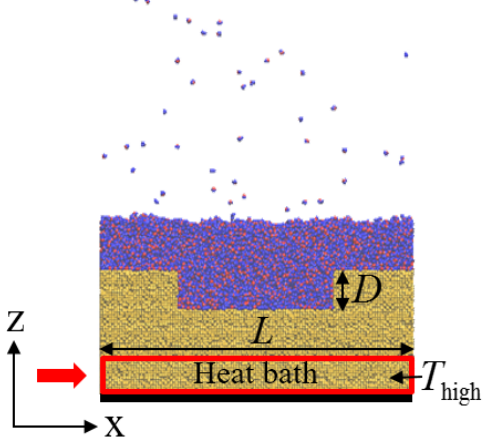

Figure 2. (a) Schematic of an evaporating thin film on a nanostructured surface. (b) Equivalent thermal circuit. (c-d) MD simulation setups for water films evaporating on (c) a flat and (d) a square nanostructured gold surface.

Assuming $\left|T_{\mathrm{lv}}-T_{\mathrm{v}}\right| \ll<T_{\mathrm{v}}$ and using Eq. (3), Eq. (1) becomes

$$
q^{\prime \prime}=\left(\frac{2 \hat{\sigma} h_{f g}}{2-\hat{\sigma}}\right)\left(\frac{M}{2 \pi R T_{\mathrm{v}}}\right)^{1 / 2}\left(\frac{P_{\mathrm{v}} M}{\rho_{\mathrm{l}} R T_{\mathrm{v}}}\right)\left[\frac{\rho_{\mathrm{l}} h_{\mathrm{fg}}}{T_{\mathrm{v}}}\left(T_{\mathrm{lv}}-T_{\mathrm{v}}\right)-\left(P_{\mathrm{C}}+\Pi\right)\right]
$$

Substituting Eq. (4) into Eq. (5) and using the ideal gas law, it fellows 


$$
q^{\prime \prime}=\frac{\left(\frac{2 \hat{\sigma} h_{\mathrm{fg}}}{2-\hat{\sigma}}\right)\left(\frac{M}{2 \pi R T_{\mathrm{v}}}\right)^{1 / 2}\left(\frac{\rho_{\mathrm{v}}}{\rho_{1}}\right)\left[\frac{\rho_{l} h_{\mathrm{fg}}}{T_{\mathrm{v}}}\left(T_{\mathrm{s}}-T_{\mathrm{v}}\right)-\left(P_{C}+\Pi\right)\right]}{1+\left(\frac{2 \hat{\sigma} h_{f g}}{2-\hat{\sigma}}\right)\left(\frac{M}{2 \pi R T_{\mathrm{v}}}\right)^{1 / 2}\left(\frac{\rho_{\mathrm{v}} h_{\mathrm{fg}}}{T_{\mathrm{v}}}\right)\left(\frac{\delta^{*}}{k_{1}}+R_{\mathrm{K}}\right)}
$$

The heat transfer coefficient, $h=q^{\prime \prime} /\left(T_{\mathrm{s}}-T_{\mathrm{v}}\right)$, of thin film evaporation is hence given by

$$
h=\frac{\left(\frac{2 \hat{\sigma} h_{\mathrm{fg}}}{2-\hat{\sigma}}\right)\left(\frac{M}{2 \pi R T_{\mathrm{v}}}\right)^{1 / 2}\left(\frac{\rho_{\mathrm{v}}}{\rho_{\mathrm{l}}}\right)\left[-\frac{P_{C}+\Pi}{\left(T_{\mathrm{s}}-T_{\mathrm{v}}\right)}+\frac{\rho_{l} h_{\mathrm{fg}}}{T_{\mathrm{v}}}\right]}{1+\left(\frac{2 \hat{\sigma} h_{f g}}{2-\hat{\sigma}}\right)\left(\frac{M}{2 \pi R T_{\mathrm{v}}}\right)^{1 / 2}\left(\frac{\rho_{\mathrm{v}} h_{\mathrm{fg}}}{T_{\mathrm{v}}}\right)\left(\frac{\delta^{*}}{k_{\mathrm{l}}}+R_{\mathrm{K}}\right)}
$$

Averaging along the solid surface (in the $x$ direction), the averaged heat transfer coefficient can be calculated by

$$
\bar{h}=\frac{1}{L} \int_{-L / 2}^{L / 2} h(x) d x
$$

where the temperatures of both the solid surface $T_{\mathrm{s}}$ and vapor $T_{\mathrm{v}}$ are assumed to be constant, but the disjoining pressure, $\Pi$, capillary pressure, $P_{\mathrm{C}}$, and modified local film thickness, $\delta^{*}$, are all functions of location $x$.

For cases where the van der Waals forces are the dominant intermolecular interactions between the liquid and solid surface, Hu et al. developed a closed-form model to predict the disjoining pressure of a thin liquid film on a 2D periodic nanostructured surface as [29]

$$
\Pi(x)=\frac{A r^{3}}{6 \pi\left[\zeta_{L}(x)-\zeta_{S}(x)\right]^{3}}
$$

where $A$ is the Hamaker constant. And the capillary pressure is given as

$$
P_{\mathrm{C}}(x)=\frac{\gamma \zeta_{\mathrm{L}}^{\prime \prime}(x)}{\sqrt{1+\left[\zeta_{\mathrm{L}}^{\prime}(x)\right]^{2}}}
$$


where $\gamma$ is the surface tension of the liquid. The meniscus shape, $\zeta_{L}(x)$, can be represented by a Fourier cosine series

$$
\zeta_{\mathrm{L}}(x)=\sum_{n=1}^{\infty} a_{\mathrm{n}} \cos \left(\frac{2 \pi n}{L} x\right)+\delta_{0}
$$

Here, the Fourier coefficients, $a_{\mathrm{n}}$, is determined by

$$
\begin{gathered}
\int_{-L / 2}^{L / 2} \frac{\frac{2 \pi n}{L} \sin \left(\frac{2 \pi n}{L} x\right) \sum_{m=1}^{\infty} a_{\mathrm{m}} \frac{2 \pi m}{L} \sin \left(\frac{2 \pi m}{L} x\right) d x}{\sqrt{1+\left[\sum_{m=1}^{\infty} a_{\mathrm{m}} \frac{2 \pi m}{L} \sin \left(\frac{2 \pi m}{L} x\right)\right]^{2}}} \\
-\frac{A r^{2}}{6 \pi \gamma} \int_{-L / 2}^{L / 2} \frac{\cos \left(\frac{2 \pi n}{L} x\right) d x}{\left[\sum_{m=1}^{\infty} a_{\mathrm{m}} \cos \left(\frac{2 \pi m}{L} x\right)+\delta_{0}-\zeta_{\mathrm{S}}(x)\right]^{3}}=0
\end{gathered}
$$

With a known Kapitza resistance, Eqs. (7)-(12) can be used to calculate the averaged heat transfer coefficient of an evaporating thin liquid film on any given two-dimensional periodic nanostructure.

It has been shown that [29], for a liquid film thickness much smaller than the nanostructure depth, $\delta_{0} \ll D$, the meniscus is conformal to the solid nanostructure with a constant local film thickness, namely, $\delta^{*}(x) \equiv \delta_{0} / r$, and the disjoining pressure can be simplified as

$$
\bar{\Pi}=\frac{A r^{3}}{6 \pi \delta_{0}^{3}}
$$

In the limit of $\delta_{0} \ll D$, the averaged heat transfer coefficient of an evaporating thin film on a nanostructured surface is hence given by 


$$
\bar{h}=\frac{\left(\frac{2 \hat{\sigma} h_{\mathrm{fg}}}{2-\hat{\sigma}}\right)\left(\frac{M}{2 \pi R T_{\mathrm{v}}}\right)^{1 / 2}\left(\frac{\rho_{\mathrm{v}}}{\rho_{1}}\right)\left[-\frac{A r^{3}}{6 \pi \delta_{0}^{3}\left(T_{\mathrm{s}}-T_{\mathrm{v}}\right)}+\frac{\rho_{l} h_{\mathrm{fg}}}{T_{\mathrm{v}}}\right]}{1+\left(\frac{2 \hat{\sigma} h_{f g}}{2-\hat{\sigma}}\right)\left(\frac{M}{2 \pi R T_{\mathrm{v}}}\right)^{1 / 2}\left(\frac{\rho_{\mathrm{v}} h_{\mathrm{fg}}}{T_{\mathrm{v}}}\right)\left(\frac{\delta_{0}}{r k_{1}}+R_{\mathrm{K}}\right)}
$$

where the capillary pressure cancels out due to symmetry.

Note that the Kapitza resistance, $R_{\mathrm{K}}$, is a strong function of the nanostructures [32, 33]. Here, we use a semi-empirical model to account for the effect of nanostructures on Kapitza resistance. Both experiments and atomic simulations suggest a linear relation between the interfacial thermal conductance (i.e., $R_{\mathrm{K}}^{-1}$ ) and the solid-liquid interaction energy per unit area $[31,39,40]$ that scales linearly with the Wenzel roughness ratio, following $R_{\mathrm{K}}^{-1} \propto r$. By fitting the MD results for the Kapitza resistance at a water-gold interface [32], it follows

$$
R_{\mathrm{K}}^{-1}=(2.282 r-1.183) \times 10^{8} \mathrm{~W} / \mathrm{m}^{2} \mathrm{~K}
$$

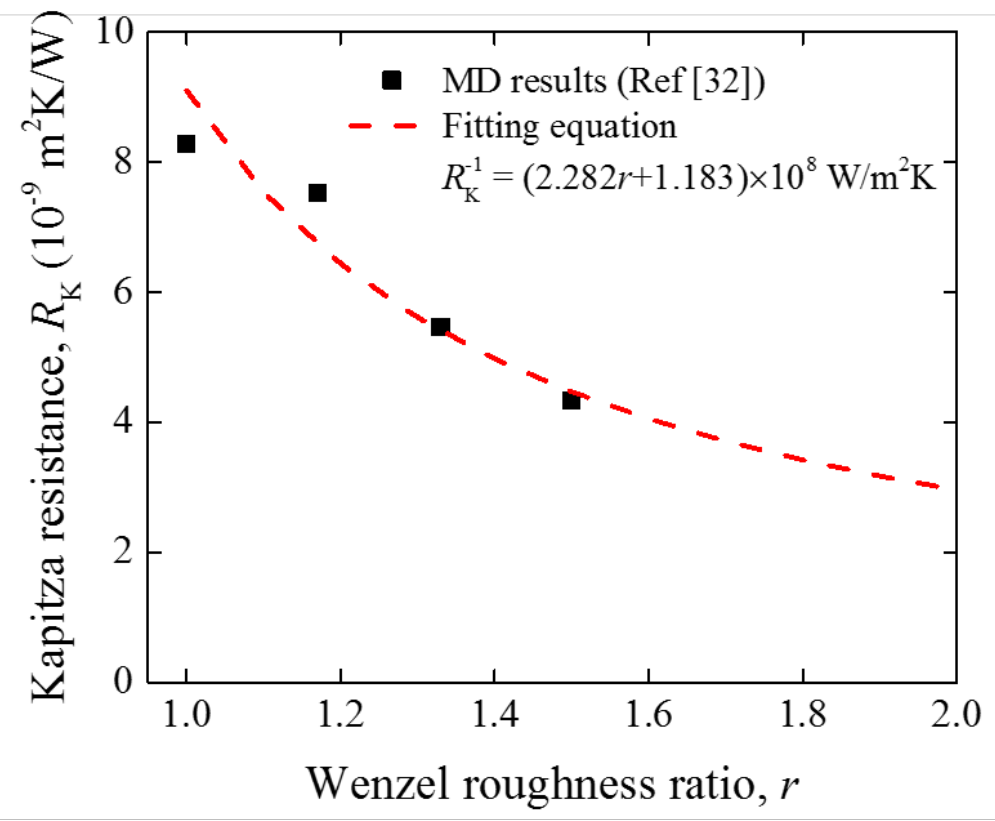


Figure 3. MD calculated [32] and fitting of Kapitza resistance at a water-gold interface as a function of the Wenzel roughness ratio.

\section{Molecular dynamic simulations}

MD simulations were performed for evaporation of water thin films on flat and square nanostructured gold substrates based on the same geometrical parameters as used in the closedform model. Figures $2 \mathrm{c}$ and $2 \mathrm{~d}$ show the simulation setups for a water film of thickness $\delta_{0}$ on flat and square nanostructured gold surfaces, respectively. In each setup, two water films were separated 20nm apart. Periodic boundary conditions were applied in all directions and two atomic layers of gold were frozen at each end in the $\mathrm{z}$ direction. A 12-6 Lennard-Jones potential was used to describe the water-gold interactions, where the energy and distance parameters are $\varepsilon_{\mathrm{Au} / \mathrm{O}}=8.3 \mathrm{~kJ} / \mathrm{mol}, \sigma_{\mathrm{Au} / \mathrm{O}}=2.95 \AA, \varepsilon_{\mathrm{Au} / \mathrm{H}}=0$ and $\sigma_{\mathrm{Au} / \mathrm{H}}=0$ [32]. The embedded atom model (EAM) potential [41] was used to model the interaction between gold atoms and the TIP4P-Ew water model that has been shown to accurately describe liquid-vapor coexistent was used for water [42]. The long-range intermolecular forces are calculated using the particle-particle particle-mesh (PPPM) method. The Hamaker constant was calculated based on the LennardJones potential for water-gold interactions from $A=4 \pi^{2} \rho_{l} \rho_{s} \sigma_{\mathrm{Au} / \mathrm{O}}^{6} \varepsilon_{\mathrm{Au} / \mathrm{O}}$ [43], where $\rho_{l}$ and $\rho_{s}$ are the densities of water and gold, respectively. All simulations were performed using LAMMPS [44] with a time step of $1 \mathrm{fs}$. The wavelength of the nanostructure, $L$, was fixed at $22.84 \mathrm{~nm}$. The nanostructure depths $(D)$ of $2.85 \mathrm{~nm}, 5.71 \mathrm{~nm}$ and $11.42 \mathrm{~nm}$ were considered corresponding to nanostructure aspect ratios $(2 D / L)$ of $1: 4,1: 2$ and $1: 1$, respectively. Thin water films with thicknesses of $1.25 \mathrm{~nm}, 2.48 \mathrm{~nm}, 3.72 \mathrm{~nm}, 4.75 \mathrm{~nm}$ and $9.92 \mathrm{~nm}$ were simulated on flat gold surface and square nanostructured gold surfaces with depths of $2.85 \mathrm{~nm}$ and $5.71 \mathrm{~nm}$, 
and films with thickness of $1.9 \mathrm{~nm}, 3.1 \mathrm{~nm}, 6.2 \mathrm{~nm}$ and $12.4 \mathrm{~nm}$ were simulated on square nanostructured gold surfaces with depth of $11.42 \mathrm{~nm}$.

The simulation system was first equilibrated in an NVT (with N being the number of atoms, $\mathrm{V}$ the volume, and $\mathrm{T}$ the temperature) ensemble at $370.3 \mathrm{~K}$ (i.e., the boiling point of the TIP4PEw water model, $T_{\mathrm{b}}$ ) [42]. Once equilibrated, the non-equilibrium molecular dynamics (NEMD) simulations in an NVE ( $\mathrm{E}$ is the total energy) ensemble were used to compute the heat transfer rate across the entire water-gold system in $z$. As illustrated in the MD setups in Fig 2c and 2d, the heat bath and cold bath were kept at temperatures $T_{\text {high }}\left(T_{\text {high }}>T_{\mathrm{b}}\right)$ and $T_{\text {low }}\left(T_{\text {low }}<T_{\mathrm{b}}\right)$, respectively. In all cases, symmetric heating and cooling were used, i.e. $T_{\text {high }}-T_{\mathrm{b}}=T_{\mathrm{b}}-T_{\text {low }}$. Four superheat values $\left(T_{\text {high }}-T_{\mathrm{b}}\right)$ of $25 \mathrm{~K}, 50 \mathrm{~K}, 75 \mathrm{~K}$, and $100 \mathrm{~K}$ were used at the heat bath. As heat conducts through the solid wall to evaporate the water molecules on the solid surface, the evaporated water molecules travel across the vapor phase and then condense on the surface of the cold wall. The evaporation and condensation rates were evaluated using the change in the number of water molecules per unit time in thin films on the hot and cold walls, respectively. The evaporation flux, $n^{\prime \prime}$, was then calculated by dividing the evaporation rate with the normal area of the MD setup (i.e. $L_{\mathrm{x}} L_{\mathrm{y}}$ ). Here the calculated evaporation flux was the averaged value along the $x$ direction, while the variation along $x$ was not considered. The system reaches a steady state when the averaged evaporation flux becomes constant, $n_{\mathrm{s}}^{\prime \prime}$, and the steady state heat flux was calculated as $\bar{q}^{\prime \prime}=n_{\mathrm{s}}^{\prime \prime} h_{\mathrm{fg}}$. The temperature profiles along the $z$-axis (i.e., the heat transfer direction) of a water-gold system were calculated at steady state, where the simulation setups were divided into slices with thickness of $0.2 \mathrm{~nm}$ each in $z$ where the temperatures in each slice 
were calculated. In the temperature calculation, the center-of-mass velocity was subtracted from each atom [45].

\section{Results and Discussion}

Figure 4a shows the MD-calculated averaged evaporation flux, $n^{\prime \prime}$, as a function of time for a water thin film of thickness $2.48 \mathrm{~nm}$ evaporating on a square nanostructured gold surface of $2.85 \mathrm{~nm}$ deep with $100 \mathrm{~K}$ superheat at the heat bath. Immediately after applying heat to the system to fix the temperature difference across the hot and cold baths at $t=0$, the evaporation flux increases rapidly until $t \sim 400 \mathrm{ps}$ when the evaporation flux plateaus and fluctuates around the steady-state value of $n_{\mathrm{s}}^{\prime \prime}=3.57 \times 10^{4} \mathrm{~mol} / \mathrm{m}^{2} \mathrm{~s}$, which is a function of the film thickness and nanostructure depth. As evaporation continues, the thin film thickness on the heated surface decreases, leading to a stronger disjoining pressure. At $t \sim 2 \mathrm{~ns}$, the disjoining pressure becomes strong enough to suppress evaporation such that the evaporation flux starts to decrease. After $t>$ $4.5 \mathrm{~ns}$, the disjoining pressure is so strong that there is barely any water molecule evaporating and the evaporation flux is around zero. Figure $4 \mathrm{~b}$ shows the corresponding snapshots at $t=0$, $1.5 \mathrm{~ns}, 3.0 \mathrm{~ns}$ and $4.5 \mathrm{~ns}$. It is clear that a thin non-evaporating film remains on the heated surface at $4.5 \mathrm{~ns}$ when evaporation completely stops.

Figure 5 shows MD-calculated steady-state temperature profiles along $z$ of a water thin film with thickness of $4.5 \mathrm{~nm}$ on a square nanostructured surface with depth of $2.85 \mathrm{~nm}$ under bath superheat $\left(T_{\text {high }}-T_{\mathrm{b}}\right)$ of $25 \mathrm{~K}, 50 \mathrm{~K}, 75 \mathrm{~K}$ and $100 \mathrm{~K}$. The wall temperature $T_{\mathrm{s}}$ is obtained by linear extrapolating the temperature profile of solid at the solid-liquid interface for cases with flat surface, or by averaging the temperature of solid wall in the nanostructured region where the solid temperature co-exists with the liquid temperature for cases with nanostructures. It can be 
observed from Fig. 5 that, the liquid temperature decreases along $z$ due to the conduction resistance in the liquid. A temperature discontinuity is observed at the liquid-vapor interface due to the evaporation resistance, and the temperature in the vapor region is very uniform. By averaging the local temperatures in the vapor phase, the vapor temperature, $T_{\mathrm{v}}$, is determined.

Defining the wall superheat as $\Delta T_{\text {wall }}=T_{\mathrm{s}}-T_{\mathrm{v}}$, the heat transfer coefficient of thin film evaporation is calculated using $\bar{h}=\vec{q} / \Delta T_{\text {wall }}$.

(a)

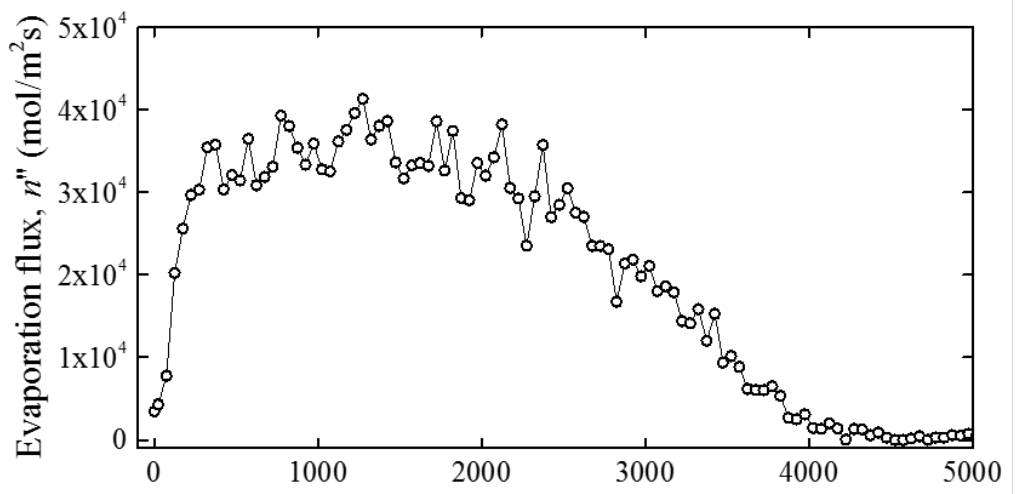

(b)

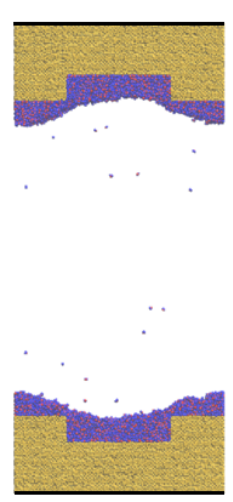

$t=0$
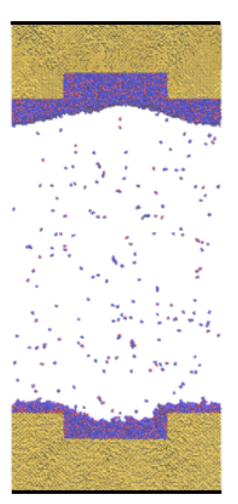

$t=1.5 \mathrm{~ns}$

Time, $t$ (ps)
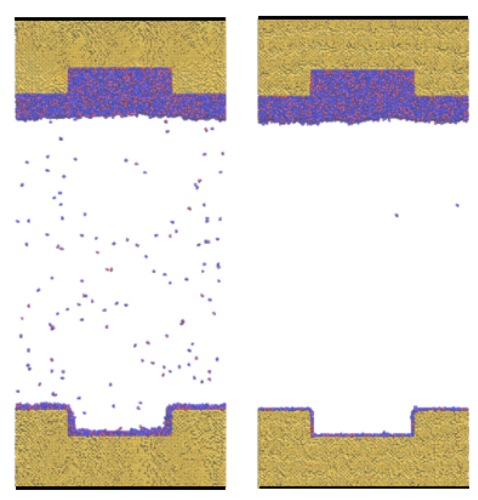

$t=3 \mathrm{~ns}$

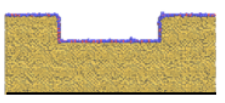

$t=4.5 \mathrm{~ns}$

Figure 4. (a) MD-calculated evaporation flux of a water film on a square nanostructured surface as a function of time. (b) Snapshots of a water thin film evaporating on a square nanostructured surface at $t=1,1.5,3$, and $4.5 \mathrm{~ns}$. 


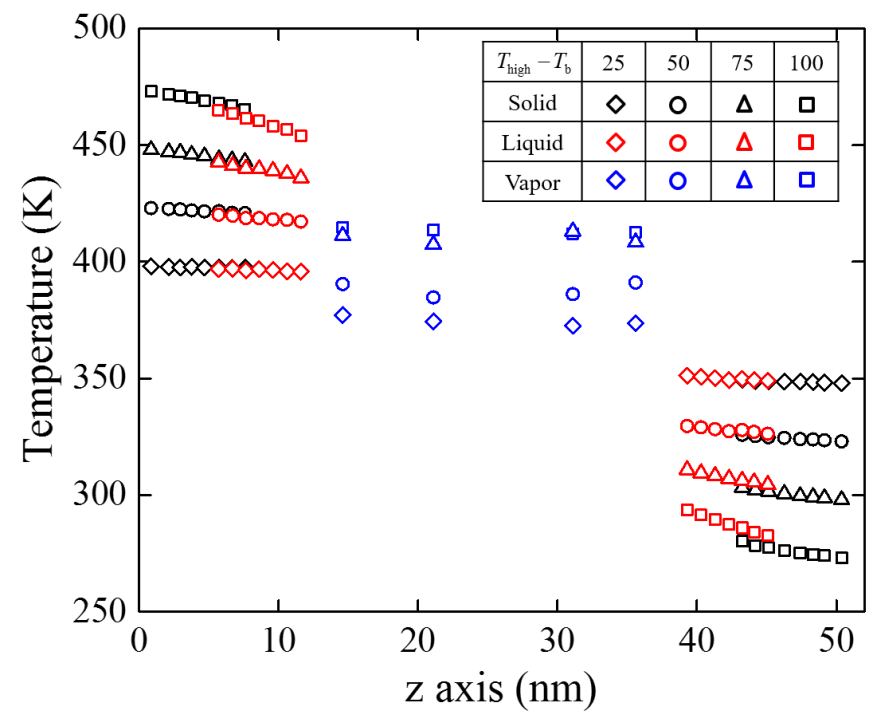

Figure 5. MD-simulated steady-state temperature profiles along $z$ of a water thin film with thickness of $4.5 \mathrm{~nm}$ on a square nanostructured surface with depth of $2.85 \mathrm{~nm}$ under $25 \mathrm{~K}, 50 \mathrm{~K}$, $75 \mathrm{~K}$ and $100 \mathrm{~K}$ of superheat $\left(T_{\text {high }}-T_{\mathrm{b}}\right)$.

Figure 6 shows the MD-calculated and model-predicted averaged heat transfer coefficient, $\bar{h}$, as a function of film thickness for a water film evaporating on flat and square nanostructured surfaces of depths $D=2.85 \mathrm{~nm}, 5.71 \mathrm{~nm}$ and $11.42 \mathrm{~nm}$ under bath superheat of $T_{\text {high }}-T_{\mathrm{b}}=100$ $\mathrm{K}$. The solid and dashed lines represent model predictions using the complete closed-form model of Eq. (8) and the simplified model of Eq. (14), respectively, and the symbols are for MD results. The accommodation coefficient of evaporation is assumed to be $\hat{\sigma}=1.0$ [46] in all model predictions. The results show a less than 5\% deviation between the closed-form and simplified models, both in good agreements with the MD results. It is noted that due to numerical instabilities while calculating the Fourier coefficients, the closed-form model becomes unstable 
at small liquid film thicknesses, i.e., $1.1 \mathrm{~nm}$ for $D=2.85 \mathrm{~nm}, 1.9 \mathrm{~nm}$ for $D=5.71 \mathrm{~nm}$, and 9.8 nm for $D=11.42 \mathrm{~nm}$, respectively. As shown in Fig. 6, for each nanostructure depth, the heat transfer coefficient first increases and then decreases with the film thickness: there exists a critical film thickness, $\delta_{\text {crit }}$, where the heat transfer coefficient reaches its maximum value, $\bar{h}_{\max }$. Recall that in the equivalent thermal circuit model shown in Fig. 2b, the heat transfer coefficient of an evaporating thin film is a function of the evaporation resistance, conduction resistance, and the Kapitza resistance. While the Kapitza resistance is independent of the thin film thickness, both the evaporation resistance and the conduction resistance are functions of the film thickness. As the film thickness increases, the disjoining pressure and its suppression on evaporation decrease, leading to a smaller evaporation resistance. On the other hand, a thicker film leads to a larger conduction resistance. The overall effect of film thickness on the heat transfer coefficient is the result of the competition between the disjoining pressure and conduction. At the critical film thickness, $\delta_{\text {crit }}$, the increase in the conduction resistance is balanced with the decrease of the evaporation resistance. As for a film thickness below $\delta_{\text {crit }}$, the disjoining pressure is very strong and suppresses evaporation, and the evaporation resistance dominates. As a result, the heat transfer coefficient of an evaporating film increases with the film thickness for $\delta_{0}<\delta_{\text {crit }}$. As for a film thickness greater than $\delta_{\text {crit }}$, the conduction resistance dominates, and the heat transfer coefficient decreases with film thickness.

The effect of nanostructures on heat transfer coefficient of an evaporating meniscus is more complicated because evaporation, conduction, and Kapitza resistances are all strong functions of the nanostructures. We [32] as well as others [33] have shown that the Kapitza resistance decreases with nanostructure depth due to the enhanced solid-liquid interaction energy. As a 
result, the maximum heat transfer coefficient, $\bar{h}_{\max }$, of an evaporating meniscus increases with the nanostructure depth $D$, as shown in Fig. 6. As the disjoining pressure increases with the nanostructure depth [29], the evaporation resistance also increases with $D$, but the conduction resistance decreases with $D$ due to a smaller local film thickness. As a result, as $D$ increases, the critical film thickness, $\delta_{\text {crit }}$, also increases. The critical film thicknesses estimated using the simplified model, Eq. (14), are $1.61 \mathrm{~nm}, 1.98 \mathrm{~nm}, 2.35 \mathrm{~nm}$, and $3.10 \mathrm{~nm}$ for $D=0$ (flat surface), $2.85 \mathrm{~nm}, 5.71 \mathrm{~nm}$, and $11.42 \mathrm{~nm}$, respectively. Furthermore, when the conduction resistance dominates (i.e., $\delta_{0}>\delta_{\text {crit }}$ ), the heat transfer coefficient of an evaporating thin film increases with nanostructure depth.

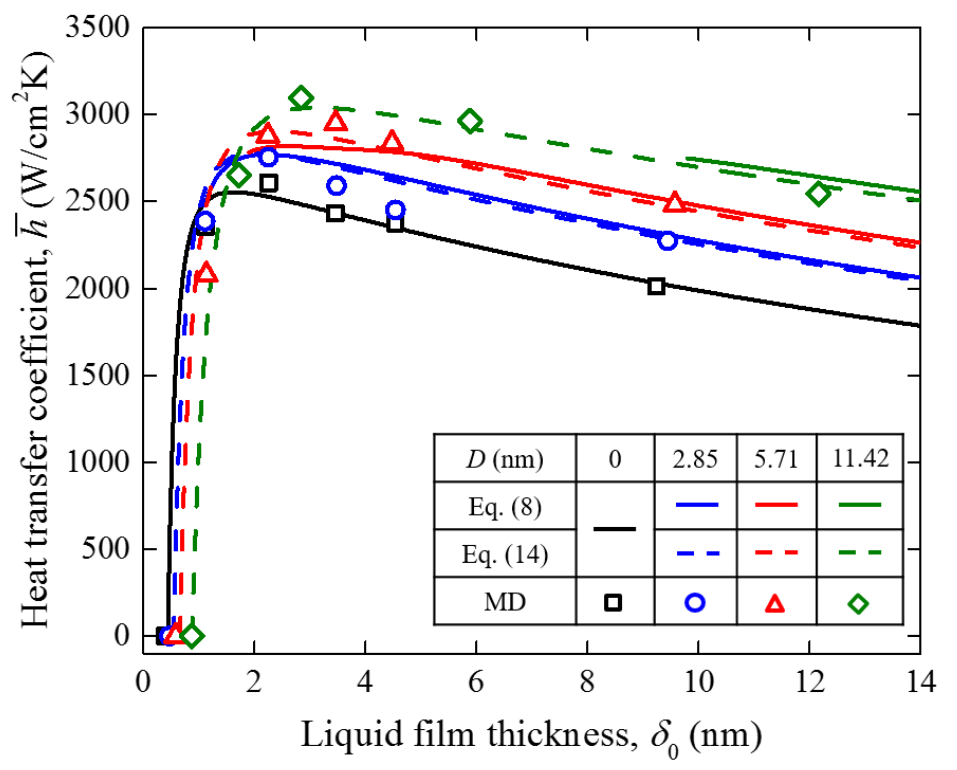

Figure 6. Comparison between MD-calculated and model-predicted heat transfer coefficients as a function of the liquid film thickness and nanostructure depth for a water thin film evaporating on flat and square nanostructured gold surfaces. 
By setting $d \bar{h} /\left.d \delta_{0}\right|_{\delta_{0}=\delta_{\text {crit }}}=0$ and using Eq. (14), the critical film thickness, $\delta_{\text {crit }}$, where the maximum heat transfer coefficient is reached can be determined using

$$
\delta_{\text {crit }}^{4}+B \delta_{\text {crit }}+C=0
$$

where the constants $B$ and $C$ are given as $B=-\frac{4 A r^{3} T_{\mathrm{v}}}{6 \pi \rho_{\mathrm{l}} h_{\mathrm{fg}}\left(T_{\mathrm{s}}-T_{\mathrm{v}}\right)}$ which scales with $r^{3}$, and $C=-\frac{3 A r^{4} T_{\mathrm{v}} k_{1}}{6 \pi \rho_{1} h_{\mathrm{fg}}\left(T_{\mathrm{s}}-T_{\mathrm{v}}\right)}\left(R_{\mathrm{K}}+\left(\frac{2-\hat{\sigma}}{2 \hat{\sigma} h_{\mathrm{fg}}^{2}}\right)\left(\frac{2 \pi R T_{\mathrm{v}}}{M}\right)^{1 / 2} \frac{T_{\mathrm{v}}}{\rho_{1}}\right)$. Note that, the Kapitza resistance reaches its maximum value on a flat surface (i.e., $R_{\mathrm{K}}=R_{\mathrm{K} 0}$ for $r=1$ ), decreases with $r$, and finally converges to 0 at $r \rightarrow \infty$. When the Kapitza resistance is negligible (i.e., $R_{\mathrm{K}}=0$ ), $C$ converges to $-\frac{3 A r^{4} T_{\mathrm{v}} k_{1}}{6 \pi \rho_{\mathrm{l}} h_{\mathrm{fg}}\left(T_{\mathrm{s}}-T_{\mathrm{v}}\right)}\left(\left(\frac{2-\hat{\sigma}}{2 \hat{\sigma} h_{\mathrm{fg}}^{2}}\right)\left(\frac{2 \pi R T_{\mathrm{v}}}{M}\right)^{1 / 2} \frac{T_{\mathrm{v}}}{\rho_{\mathrm{l}}}\right)$ which scales with $r^{4}$. Thus, using Eq. (16), $\delta_{\text {crit }}$ scales linearly with $r$. Similarly, when $R_{\mathrm{K}}=R_{\mathrm{K} 0}$, the constant $C$ scales with $r^{4}$, such that $\delta_{\text {crit }}$ increases linearly with $r$ based on Eq. (16). Note that, the $\delta_{\text {crit }}$ calculated at $R_{\mathrm{K}}=R_{\mathrm{K} 0}$ and $R_{\mathrm{K}}=0$ sets its upper and lower bounds. In addition, by substituting $\delta_{\text {crit }}$ in Eq. (14), the maximum heat transfer coefficient, $\bar{h}_{\max }$, follows

$$
\bar{h}_{\max }=\frac{\left(\frac{2 \hat{\sigma} h_{\mathrm{fg}}}{2-\hat{\sigma}}\right)\left(\frac{M}{2 \pi R T_{\mathrm{v}}}\right)^{1 / 2}\left(\frac{\rho_{\mathrm{v}}}{\rho_{1}}\right)\left[-\frac{A r^{3}}{6 \pi \delta_{\text {crit }}^{3}\left(T_{\mathrm{s}}-T_{\mathrm{v}}\right)}+\frac{\rho_{l} h_{\mathrm{fg}}}{T_{\mathrm{v}}}\right]}{1+\left(\frac{2 \hat{\sigma} h_{f g}}{2-\hat{\sigma}}\right)\left(\frac{M}{2 \pi R T_{\mathrm{v}}}\right)^{1 / 2}\left(\frac{\rho_{\mathrm{v}} h_{\mathrm{fg}}}{T_{\mathrm{v}}}\right)\left(\frac{\delta_{\text {crit }}}{r k_{\mathrm{l}}}+R_{\mathrm{K}}\right)}
$$

Figure 7a shows the model-predicted critical film thickness as a function of Wenzel roughness ratio for a thin water film evaporating on a square nanostructured gold surface. The 
solid line represents the prediction of the closed-form model using Eq. (16), and the red and blue dashed lines represent the prediction of Eq. (16) in the limit of $R_{\mathrm{K}} \rightarrow R_{\mathrm{K} 0}$ (Kapitza resistance equals to the value of a flat surface) and $R_{\mathrm{K}} \rightarrow 0$ (Kapitza resistance is negligible), respectively. The results show that the closed-form model agrees well with the simplified equation in the limit of $R_{\mathrm{K}} \rightarrow R_{\mathrm{K} 0}$ for a small Wenzel roughness ratio $r$, and converges to the simplified model in the limit of $R_{\mathrm{K}} \rightarrow 0$ as $r$ increases. Figure $7 \mathrm{~b}$ depicts the model-predicted maximum heat transfer coefficient as a function of the Wenzel roughness ratio, where the solid line represents the prediction of the closed-form model and the red and blue lines represent the lower and upper bounds of $h_{\max }$ at $R_{\mathrm{K}} \rightarrow R_{\mathrm{K} 0}$ and $R_{\mathrm{K}} \rightarrow 0$, respectively. Note that, as the Wenzel roughness ratio increases, the Kapitza resistance decreases. As a result, the maximum heat transfer coefficient of an evaporating film increases with the Wenzel roughness ratio and reaches its upper bound in the limit of $R_{\mathrm{K}} \rightarrow 0$ at $r \rightarrow \infty$.

(a)

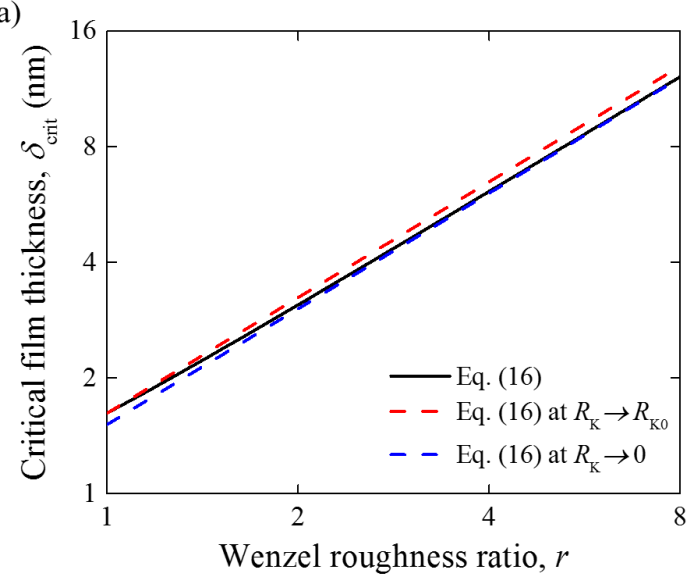

(b)

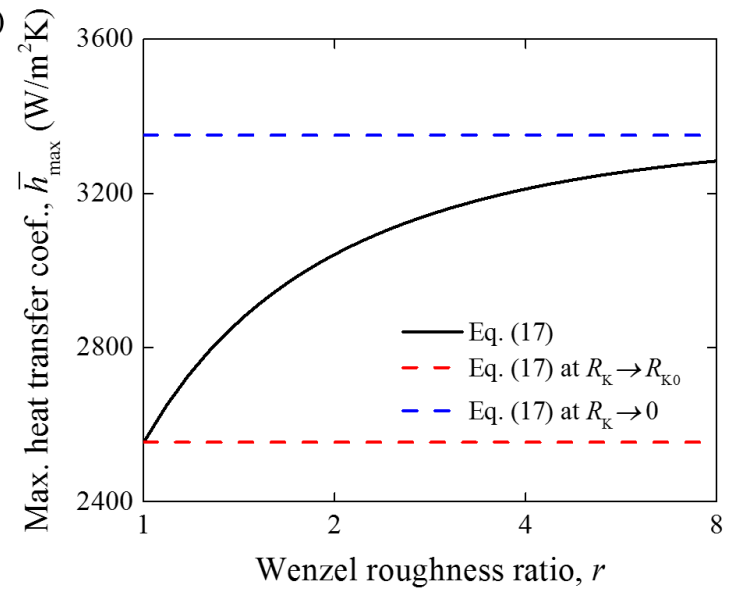

Figure 7. (a) Critical film thickness and (b) Maximum heat transfer coefficient of an evaporating film as a function of Wenzel roughness ratio. 
Figure 8 shows the comparison between the model-predicted and MD-simulated results for the scaled heat transfer coefficient of an evaporating water thin film on a square nanostructured gold surface as a function of the scaled film thickness. The black line shows the model prediction using Eq. (14). Good agreement is found between the model predictions and MD results. When $\delta_{0} / \delta_{\text {crit }}<1$, the evaporation resistance dominates the heat transfer process, and the scaled heat transfer coefficient increases with the scaled film thickness. When $\delta_{0} / \delta_{\text {crit }}>1$, the conduction resistance dominates, the scaled heat transfer coefficient decreases with the scaled film thickness.

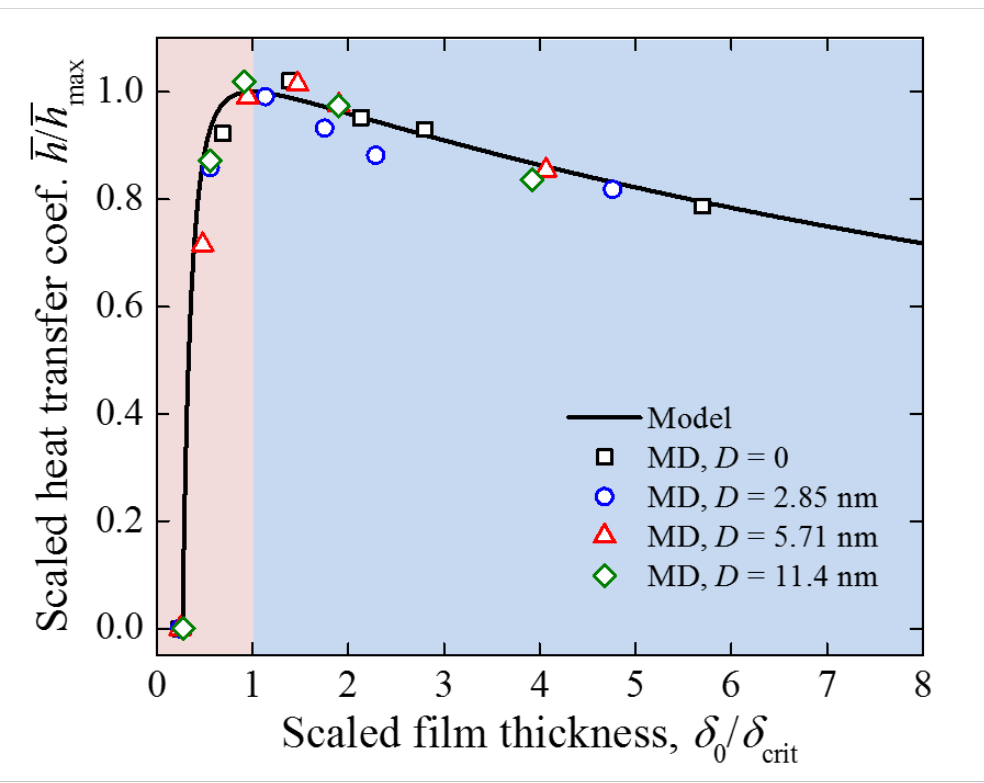

Figure 8. Comparison between MD-calculated and model-predicted scaled heat transfer coefficient of an evaporating meniscus as a function of the scaled film thickness.

The model prediction and MD simulations in the present work calculate the steady-state heat transfer coefficient of a static liquid film on nanostructures. The model is verified against MD 
results for thin film evaporation on square nanostructures, but can be applied for any other type of 2D periodic structures (e.g., triangular or cylindrical structures). The results can be used to determine the heat transfer performance assuming sufficient liquid delivery to the evaporating thin film region. In a real practice, insufficient liquid supply can lead to drying out of the thin film, limiting the heat transfer coefficient. Further work will focus on investigating the effect of nanostructures on liquid delivery. Integrating the model of the heat transfer coefficient with the liquid delivery rate will enable accurate predictions of thin film evaporation in nanostructureenhanced boiling heat transfer.

\section{Conclusion}

In this study, a closed-form model is derived to predict the heat transfer coefficient of a thin liquid film evaporating on a nanostructured surface. Molecular dynamics simulations of water thin films on square gold nanostructures are performed to verify the model. Good agreements are obtained between MD results and model predictions for thin films of varying thickness on nanostructured surfaces of varying depth. The results show that there exists a critical film thickness, $\delta_{\text {crit }}$, at which the heat transfer coefficient reaches its maximum value. For a film with thicknesses below $\delta_{\text {crit }}$, the evaporation resistance dominates the heat transfer, and heat transfer coefficient increases with film thickness and decreases with nanostructure depth. For a film with thicknesses above $\delta_{\text {crit }}$, the conduction resistance dominates the heat transfer, and heat transfer coefficient decreases with film thickness and increases with nanostructure depth. In addition, the critical film thickness increases with Wenzel roughness ratio. The maximum heat transfer coefficient also increases with Wenzel roughness ratio due to the reduction in Kapitza resistance. The model developed here will enable more accurate prediction of evaporation rate of a static 
thin liquid film on nanostructures. Future work will focus on determining the liquid delivery rate on nanostructures, which combined with the present work, will enable accurate prediction of the evaporation rate of the thin liquid films during boiling.

\section{Acknowledgements}

Support for this work was provided by the National Science Foundation (No. DMR1104835 and CMMI-1401438). Computational resources were provided by the Extreme Science and Engineering Discovery Environment (XSEDE) (Grant \#TG-CTS110056).

\section{References}

[1] R. Chen, M.C. Lu, V. Srinivasan, Z. Wang, H.H. Cho, A. Majumdar, Nanowires for Enhanced Boiling Heat Transfer, Nano Letters, 9(2) (2009) 548-553.

[2] N. Zuber, Hydrodynamic Aspects of Boiling Heat Transfer (thesis), United States Atomic Energy Commission, Technical Information Service, 1959.

[3] M.M. Rahman, E. Ölçeroğlu, M. McCarthy, Role of Wickability on the Critical Heat Flux of Structured Superhydrophilic Surfaces, Langmuir, (2014).

[4] S.G. Kandlikar, Controlling bubble motion over heated surface through evaporation momentum force to enhance pool boiling heat transfer, Applied Physics Letters, 102(5) (2013) 051611.

[5] C. Li, Z. Wang, P.I. Wang, Y. Peles, N. Koratkar, G.P. Peterson, Nanostructured copper interfaces for enhanced boiling, Small, 4(8) (2008) 1084-1088.

[6] A.R. Betz, J. Jenkins, C.-J.C. Kim, D. Attinger, Boiling heat transfer on superhydrophilic, superhydrophobic, and superbiphilic surfaces, International Journal of Heat and Mass Transfer, 57(2) (2013) 733-741.

[7] K.-H. Chu, Y. Soo Joung, R. Enright, C.R. Buie, E.N. Wang, Hierarchically structured surfaces for boiling critical heat flux enhancement, Applied Physics Letters, 102(15) (2013) 151602.

[8] X.M. Dai, F.H. Yang, R.G. Yang, X.Y. Huang, W.A. Rigdon, X.D. Li, C. Li, Biphilic nanoporous surfaces enabled exceptional drag reduction and capillary evaporation enhancement, Applied Physics Letters, 105(19) (2014).

[9] C.-K. Guan, B. Bon, J. Klausner, R. Mei, Comparison of CHF Enhancement on Microstructured Surfaces With a Predictive Model, Heat Transfer Engineering, 35(5) (2013) 452-460.

[10] V. Sathyamurthi, H.S. Ahn, D. Banerjee, S.C. Lau, Subcooled Pool Boiling Experiments on Horizontal Heaters Coated With Carbon Nanotubes, Journal of Heat Transfer, 131(7) (2009) 071501-071501.

[11] A. Zou, S.C. Maroo, Critical height of micro/nano structures for pool boiling heat transfer enhancement, Applied Physics Letters, 103(22) (2013) 221602.

[12] Y. Nam, E. Aktinol, V.K. Dhir, S. Ju, Single bubble dynamics on a superhydrophilic surface with artificial nucleation sites, International Journal of Heat and Mass Transfer, 54(7-8) (2011) 1572-1577. 
[13] H.S. Ahn, H.J. Jo, S.H. Kang, M.H. Kim, Effect of liquid spreading due to nano/microstructures on the critical heat flux during pool boiling, Applied Physics Letters, 98(7) (2011) 071908.

[14] H.S. Ahn, G. Park, J.M. Kim, J. Kim, M.H. Kim, The effect of water absorption on critical heat flux enhancement during pool boiling, Experimental Thermal and Fluid Science, 42(0) (2012) 187-195.

[15] M.-C. Lu, C.-H. Huang, C.-T. Huang, Y.-C. Chen, A modified hydrodynamic model for pool boiling CHF considering the effects of heater size and nucleation site density, International Journal of Thermal Sciences, 91 (2015) 133-141.

[16] K.-H. Chu, R. Enright, E.N. Wang, Structured surfaces for enhanced pool boiling heat transfer, Applied Physics Letters, 100(24) (2012) 241603.

[17] C.M. Patil, S.G. Kandlikar, Pool boiling enhancement through microporous coatings selectively electrodeposited on fin tops of open microchannels, International Journal of Heat and Mass Transfer, 79 (2014) 816-828.

[18] A. Jaikumar, S.G. Kandlikar, Enhanced pool boiling heat transfer mechanisms for selectively sintered open microchannels, International Journal of Heat and Mass Transfer, 88 (2015) 652-661.

[19] D.E. Kim, D.I. Yu, S.C. Park, H.J. Kwak, H.S. Ahn, Critical heat flux triggering mechanism on micro-structured surfaces: Coalesced bubble departure frequency and liquid furnishing capability, International Journal of Heat and Mass Transfer, 91 (2015) 1237-1247.

[20] D.E. Kim, S.C. Park, D.I. Yu, M.H. Kim, H.S. Ahn, Enhanced critical heat flux by capillary driven liquid flow on the well-designed surface, Applied Physics Letters, 107(2) (2015) 023903. [21] B.S. Kim, H. Lee, S. Shin, G. Choi, H.H. Cho, Interfacial wicking dynamics and its impact on critical heat flux of boiling heat transfer, Applied Physics Letters, 105(19) (2014).

[22] N.S. Dhillon, J. Buongiorno, K.K. Varanasi, Critical heat flux maxima during boiling crisis on textured surfaces, Nat Commun, 6 (2015).

[23] P.C. Wayner Jr, Y.K. Kao, L.V. LaCroix, The interline heat-transfer coefficient of an evaporating wetting film, International Journal of Heat and Mass Transfer, 19(5) (1976) 487492.

[24] K. Park, K.-J. Noh, K.-S. Lee, Transport phenomena in the thin-film region of a microchannel, International Journal of Heat and Mass Transfer, 46(13) (2003) 2381-2388.

[25] S. Narayanan, A.G. Fedorov, Y.K. Joshi, Interfacial Transport of Evaporating Water Confined in Nanopores, Langmuir, 27(17) (2011) 10666-10676.

[26] C. Yan, H.B. Ma, Analytical Solutions of Heat Transfer and Film Thickness in Thin-Film Evaporation, Journal of Heat Transfer, 135(3) (2013) 031501-031501.

[27] H. Wang, S.V. Garimella, J.Y. Murthy, Characteristics of an evaporating thin film in a microchannel, International Journal of Heat and Mass Transfer, 50(19-20) (2007) 3933-3942.

[28] H. Wang, S.V. Garimella, J.Y. Murthy, An analytical solution for the total heat transfer in the thin-film region of an evaporating meniscus, International Journal of Heat and Mass Transfer, 51(25-26) (2008) 6317-6322.

[29] H. Hu, C.R. Weinberger, Y. Sun, Effect of Nanostructures on the Meniscus Shape and Disjoining Pressure of Ultrathin Liquid Film, Nano Letters, 14(12) (2014) 7131-7137.

[30] H. Hu, C.R. Weinberger, Y. Sun, Model of Meniscus Shape and Disjoining Pressure of Thin Liquid Films on Nanostructured Surfaces with Electrostatic Interactions, The Journal of Physical Chemistry C, 119(21) (2015) 11777-11785.

[31] H. Harikrishna, W.A. Ducker, S.T. Huxtable, The influence of interface bonding on thermal transport through solid-liquid interfaces, Applied Physics Letters, 102(25) (2013) 251606.

[32] H. Hu, Y. Sun, Effect of nanopatterns on Kapitza resistance at a water-gold interface during boiling: A molecular dynamics study, Journal of Applied Physics, 112(5) (2012) 053508053506.

[33] Y. Wang, P. Keblinski, Role of wetting and nanoscale roughness on thermal conductance at liquid-solid interface, Applied Physics Letters, 99(7) (2011).

[34] S.C. Maroo, J.N. Chung, A Possible Role of Nanostructured Ridges on Boiling Heat

Transfer Enhancement, Journal of Heat Transfer, 135(4) (2013) 041501-041501. 
[35] V.P. Carey, Liquid Vapor Phase Change Phenomena: An Introduction to the Thermophysics of Vaporization and Condensation Processes in Heat Transfer Equipment, Second Edition, Taylor \& Francis, 2007.

[36] R.W. Schrage, A theoretical study of interphase mass transfer, Columbia University Press, 1953.

[37] A. Faghri, Heat Pipe Science And Technology, Taylor \& Francis, 1995.

[38] M.O. Robbins, D. Andelman, J.-F. Joanny, Thin liquid films on rough or heterogeneous solids, Phys Rev A, 43(8) (1991) 4344-4354.

[39] Z.B. Ge, D.G. Cahill, P.V. Braun, Thermal conductance of hydrophilic and hydrophobic interfaces, Physical Review Letters, 96(18) (2006).

[40] N. Shenogina, R. Godawat, P. Keblinski, S. Garde, How Wetting and Adhesion Affect

Thermal Conductance of a Range of Hydrophobic to Hydrophilic Aqueous Interfaces, Physical Review Letters, 102(15) (2009).

[41] M.S. Daw, S.M. Foiles, M.I. Baskes, The Embedded-Atom Method - a Review of Theory and Applications, Mater Sci Rep, 9(7-8) (1993) 251-310.

[42] H.W. Horn, W.C. Swope, J.W. Pitera, Characterization of the TIP4P-Ew water model:

Vapor pressure and boiling point, Journal of Chemical Physics, 123(19) (2005).

[43] H. Hu, Y. Sun, Molecular dynamics simulations of disjoining pressure effect in ultra-thin water film on a metal surface, Appl Phys Lett, 103(26) (2013) 263110.

[44] S. Plimpton, Fast Parallel Algorithms for Short-Range Molecular-Dynamics, Journal of Computational Physics, 117(1) (1995) 1-19.

[45] G. Nagayama, M. Kawagoe, A. Tokunaga, T. Tsuruta, On the evaporation rate of ultra-thin liquid film at the nanostructured surface: A molecular dynamics study, International Journal of Thermal Sciences, 49(1) (2010) 59-66.

[46] J. Julin, M. Shiraiwa, R.E.H. Miles, J.P. Reid, U. Pöschl, I. Riipinen, Mass Accommodation of Water: Bridging the Gap Between Molecular Dynamics Simulations and Kinetic

Condensation Models, The Journal of Physical Chemistry. a, 117(2) (2013) 410-420. 